PROCEEDINGS OF THE

AMERICAN MATHEMATICAL SOCIETY

Volume 133, Number 2, Pages 331-341

S 0002-9939(04)07713-5

Article electronically published on September 16, 2004

\title{
ALGEBRAIC FUNCTIONS WITH EVEN MONODROMY
}

\author{
MICHELA ARTEBANI AND GIAN PIETRO PIROLA \\ (Communicated by Michael Stillman)
}

\begin{abstract}
Let $X$ be a compact Riemann surface of genus $g$ and $d \geq 12 g+4$ be an integer. We show that $X$ admits meromorphic functions with monodromy group equal to the alternating group $A_{d}$.
\end{abstract}

\section{Introduction}

Let $X$ be a compact Riemann surface of genus $g$ and $f: X \rightarrow \mathbb{P}^{1}$ be a meromorphic function of degree $d$. The function field $C(X)$ is a finite algebraic extension of degree $d$ of $C\left(\mathbb{P}^{1}\right)=\mathbb{C}(x)$. The monodromy group $M(f)$ is the Galois group associated to the Galois closure of the extension $C(X) / \mathbb{C}(x)$. The group $M(f)$ has a natural transitive representation in the symmetric group $S_{d}$. In this paper, we prove the existence of meromorphic functions on $X$ with even monodromy. This means that the monodromy group is contained in the alternating group $A_{d}$ on $d$ elements. In fact, we study the case:

$$
M(f)=A_{d} .
$$

The problem of finding Riemann surfaces with given monodromy group is a classical one (see for example [5]). In particular, a difficult question is that of determining all possible monodromy groups for the generic Riemann surface of genus $g$. Several aspects of this problem were considered by Zariski in [22]. Notice that the definition of monodromy group can be extended to any holomorphic map between compact Riemann surfaces. In fact, Zariski observed that there are no holomorphic maps from the generic Riemann surface of genus $g>1$ to one of positive genus. Thus the critical case to study is that of meromorphic functions. Besides, he proved that the monodromy groups of a generic Riemann surface of genus $g>6$ are not solvable. Zariski also introduced the reduction to the primitive case. This means that we can consider only minimal extensions of $\mathbb{C}(x)$ or, equivalently, indecomposable meromorphic functions.

It was first shown by Guralnick and Thompson in [13] that there are groups that cannot occur as the monodromy group of a meromorphic function on a Riemann surface of genus $g$.

Received by the editors April 17, 2003.

2000 Mathematics Subject Classification. Primary 14H05; Secondary 14H30, 14H10.

Key words and phrases. Monodromy group, spin bundle, even monodromy.

This work was partially supported by: 1) PRIN 2003: Spazi di moduli e teoria di Lie; 2) Gnsaga; 3) Far 2002 (PV): Varietá algebriche, calcolo algebrico, grafi orientati e topologici. 
Later, it was proved that the monodromy group of an indecomposable meromorphic map of degree $d$ on a generic Riemann surface of genus $g>3$ is either $S_{d}$, $d \geq(g+2) / 2$ or $A_{d}, d \geq 2 g+1$. This result is contained in a series of three papers: [11], [10] and [12].

It is well known that the symmetric group $S_{d}$ is the monodromy group of every curve of genus $g$ for $d \geq g+1$ (see [8]) and of the generic one for $d \geq(g+2) / 2$ (see [16]). As noticed by Guralnick in [9], this makes the case of the alternating group very interesting.

In a recent article, K. Magaard and H. Völklein prove that the general curve of genus $g \geq 3$ admits meromorphic functions with monodromy group $A_{d}$ and 3 -cycles (or double transpositions) as branch cycles if and only if $d \geq 2 g+1$ (see [17]).

In this paper we prove that, for $d$ sufficiently large compared to $g$, every compact Riemann surface of genus $g$ realizes the monodromy group $A_{d}$.

Theorem 1. Let $X$ be a compact Riemann surface of genus $g>0$ and $d \geq 12 g+4$ be an integer. Then there exist indecomposable meromorphic functions $f \in \mathbb{C}(X)$ of degree $d$ with $M(f)=A_{d}$. Moreover, there exists on $X$ a family $\mathcal{F}(X, d)$ of such maps with dimension:

$$
\operatorname{dim} \mathcal{F}(X, d)=\left[\frac{d+3}{2}\right]-2 g+2 .
$$

We give a brief summary of the proof, which is contained in section 4 .

We fix a spin bundle $S$ on a compact Riemann surface $X$. This is a line bundle on $X$ such that $S^{2}=K_{X}$, where $K_{X}$ is the canonical bundle. We then take a divisor $D$ on $X$ having large degree and small support (i.e. $D=n_{1} P_{1}+n_{2} P_{2}+n_{3} P_{3}$ where $P_{1}, P_{2}$ and $P_{3}$ are points of $\left.X\right)$. Let $[D]$ be the divisor given by the formal sum of points in the support of $D$. The square of a global section $s$ of $S(D)$ is a meromorphic form $\omega=s^{2}$ on $X$ with at most poles at $[D]$. It has even order at each point. If $\omega$ is exact, i.e. $\omega=d f$, then $f$ is a meromorphic function on $X$ with local monodromy in $A_{d}$ where $d=\operatorname{deg}(f)$. We call such meromorphic functions odd ramification coverings of the projective line 1 These coverings were studied in [7], 20] and [21] in connection with the spin lifting.

Given an inferior bound on the degree of $D$, the existence of nontrivial sections $s$ of $S(D)$ with exact square follows easily for dimensional reasons. In section 4 , we compute the dimension of the families of these sections when $d \geq 12 g+4$. As in the theory of special divisors (see [1]), it is possible to compute the tangent space to our families in the cohomological setting. For this, we use a rational variation of De Rham's algebraic theory and the adjustment of a technical result contained in [19] (we recall its proof in section 6). The generic smoothness of our families follows from a cohomology vanishing. Thus, comparing dimensions, we find indecomposable functions with the expected degree: their poles have maximum order at $[D]$. An algebraic lemma and a count of parameters show that the monodromy group of the general element in the family is exactly $A_{d}$.

In section 5, using the theory of admissible coverings, we show that odd ramification coverings are limits of simple odd ramification coverings, i.e. with ramification points all of order 3 with distinct images in $\mathbb{P}^{1}$. Then, Theorem 1 implies that the generic Riemann surface of genus $g$ admits simple odd ramification coverings of

\footnotetext{
${ }^{1}$ We avoid the tempting term "spin covering" from a suggestion of M. Fried who explained to us a different use of this word.
} 
degree $d \geq 12 g+4$ with monodromy group $A_{d}$ (see Theorem 2 ). This proves in a completely different way a weaker version of the result contained in [17].

In [3] S. Brivio and the second author apply Theorem 1 to give an analogous result for rational functions on complex surfaces.

\section{Preliminaries And notation}

We start recalling some known results about the monodromy group of a meromorphic function (see [18]). Let $X$ be a compact Riemann surface of genus $g$, $f: X \rightarrow \mathbb{P}^{1}$ be a meromorphic function of degree $d$ on $X$ with branch locus $B$ and ramification locus $R$. Let $p: X \backslash R \rightarrow \mathbb{P}^{1} \backslash B=Y$ be the associated topological covering. For a fixed base point $y \in Y$, the fundamental group $\Pi_{1}(Y, y)$ acts on the fibre $f^{-1}(y)$ by path lifting, giving a transitive subgroup of the symmetric group $S_{d}$. This is the monodromy group of $f$ (it is determined by $f$ up to conjugacy). In fact, the monodromy group of $f$ is isomorphic to the Galois group associated to the Galois closure of the extension $C(X) / \mathbb{C}(x)$ (see [14]).

Conversely, let us consider $B=\left\{b_{1}, \ldots, b_{r}\right\} \subset \mathbb{P}^{1}, Y=\mathbb{P}^{1} \backslash B$ and fix standard geometric generators of $\Pi_{1}(Y, y)$. Riemann's existence theorem gives a bijection between meromorphic functions of degree $d$ with branch locus $B$ (up to isomorphism) and ordered $r$-tuples of permutations $\left\{\sigma_{1}, \ldots, \sigma_{r}\right\}$ with $\sigma_{1} \cdots \sigma_{r}=i d$ and generating a transitive subgroup of $S_{d}$ (up to conjugacy).

It can easily be seen that $f$ is indecomposable if and only if its monodromy group is primitive.

We need the following group-theoretic lemmas (the first one is a classical result, the second one is just an exercise):

Lemma 1. Let $G$ be a transitive and primitive subgroup of the alternating group $A_{d}$ containing a 3 -cycle. Then $G=A_{d}$.

Lemma 2. Let $G$ be a transitive subgroup of $S_{d}$ with $g \in G$ having exactly two cycles of relatively prime length or three cycles of odd and relatively prime length. Then $G$ is a primitive subgroup.

Notation 1. Let $D$ be a divisor on $X$. We denote by $\operatorname{supp}(D)$ its support and by $[D]$ the divisor given by the formal sum of points in $\operatorname{supp}(D)$. Let $X \backslash[D]$ be the open set $X \backslash \operatorname{supp}(D)$. We write $\left[D_{1}\right] \cap\left[D_{2}\right]=\emptyset$ if $D_{1}$ and $D_{2}$ have disjoint supports. If $D_{1}-D_{2}$ is effective, we use the notation $D_{1}-D_{2} \geq 0$.

\section{SPIN SECTIONS With EXACT SQUARE}

Definition 1. Let $X$ be a compact Riemann surface. We call a line bundle $S$ on $X$ with $S^{2}=K_{X}$ a spin bundle where $K_{X}$ is the canonical bundle.

It is known (see [1]) that there are exactly $2^{2 g}$ nonequivalent spin bundles on any compact Riemann surface $X$ of genus $g$.

Fix $X$ a compact Riemann surface of genus $g, S$ a spin bundle on $X$ and $D \in$ $\operatorname{Div}(X)$ a divisor of the form:

$$
D=n_{1} P_{1}+n_{2} P_{2}+n_{3} P_{3}
$$

where $n_{1}, n_{2}, n_{3}$ are integers with $n_{1} \geq n_{2} \geq n_{3} \geq 0$ and $P_{1}, P_{2}, P_{3}$ are distinct points in $X$. Let $k$ be the degree of $[D]$. Notice that $1 \leq k \leq 3$ and $D=\sum_{i=1}^{k} n_{i} P_{i}$. We set

$$
D^{\prime}=2 D-[D] .
$$


Consider now the line bundle $S(D)$. The squares of sections $s \in H^{0}(X, S(D))$ give meromorphic forms $\omega=s^{2}$ on $X$ with at most poles in $[D]$. More precisely, their divisor is

$$
(\omega)_{0}-(\omega)_{\infty}=2 \operatorname{div}(s)-2 D=2 E,
$$

with $E=\operatorname{div}(s)-D$. If $\omega$ is an exact form with $\omega=d f$, then $f \in H^{0}\left(X, \mathcal{O}_{X}\left(D^{\prime}\right)\right)$ and

$$
\begin{aligned}
& e_{f}(x)=\operatorname{ord}_{\omega}(x)+1=2 \operatorname{ord}_{E}(x)+1 \text { if } x \in(\omega)_{0}, \\
& e_{f}(x)=-\operatorname{ord}_{\omega}(x)-1=-2 \operatorname{ord}_{E}(x)-1 \text { if } x \in(\omega)_{\infty},
\end{aligned}
$$

where $e_{f}(x)$ is the ramification index of $f$ in $x$. The map $f$ has odd ramification index in each point, hence even monodromy, i.e. $M(f)$ is contained in the alternating group. In fact, each permutation generating the monodromy group $M(f)$ can be decomposed into cycles of odd length.

Definition 2. We call a meromorphic map $f: X \rightarrow \mathbb{P}^{1}$ an odd ramification covering if all ramification points have odd index.

We define the analytic map:

$$
\Upsilon: H^{0}(X, S(D)) \rightarrow H^{1}(X \backslash[D], \mathbb{C}), \quad s \mapsto\left[s^{2}\right] .
$$

Firstly, we want to show the existence of sections $s \in H^{0}(X, S(D)), s \neq 0$ with $\Upsilon(s)=0$.

Notation 2. $h^{i}(X, L)=\operatorname{dim} H^{i}(X, L)(i=1,2) ; \mathcal{H}(X, D)=\Upsilon^{-1}(0)$;

$$
\mathcal{F}(X, D)=\left\{f \in \mathbb{C}(X): d f=s^{2}, s \in \mathcal{H}(X, D)\right\} \text {. }
$$

By the Riemann-Roch theorem and De Rham theory we have

$$
\begin{gathered}
h^{0}(X, S(D))=\operatorname{deg}(D) \text { if } \operatorname{deg}(D)>g-1 ; \\
h^{1}(X \backslash[D], \mathbb{C})=2 g+k-1
\end{gathered}
$$

where $k$ is the degree of $[D]$.

Suppose $\Upsilon(s) \neq 0$ for every $s \in H^{0}(X, S(D)) \backslash\{0\}$. This gives a contradiction if $\operatorname{deg}(D)>2 g+k-1$. Therefore we have:

Proposition 1. Let $X$ be a compact Riemann surface of genus $g$ and $D$ a divisor as in $(\star)$. If $\operatorname{deg}(D)>2 g+k-1$, then $\operatorname{dim}_{\mathbb{C}} \mathcal{H}(X, D)>0$. In particular, there exists a nonconstant odd ramification covering $f \in \mathcal{F}(X, D)$.

To get more precise information about the dimension of $\mathcal{H}(X, D)$, we are interested in the surjectivity of the differential of $\Upsilon$ in $s \in \mathcal{H}(X, D)$ :

$$
d \Upsilon(s): H^{0}(X, S(D)) \rightarrow H^{1}(X \backslash[D], \mathbb{C}) .
$$

Take the holomorphic curve $s(t)=s+t v$ in $H^{0}(X, S(D))$. Then

$$
\left[s(t)^{2}\right]=\left[s^{2}+2 t s v+t^{2} v^{2}\right]=2 t[s v]+o\left(t^{2}\right) .
$$

Therefore,

$$
d \Upsilon(s)(v)=[2 s v] .
$$

We now recall a technical lemma contained in $[19$; its proof is contained in section 6 . Let $A, B \in \operatorname{Div}(X)$ be effective divisors with $[A] \cap[B]=\emptyset, a=\operatorname{deg}[A]$ and $b=\operatorname{deg}[B]$. Consider the map:

$$
\mathcal{D}: H^{0}(X, \Omega(A-B)) \rightarrow H^{1}(X \backslash[A], \mathbb{C}), \quad \omega \mapsto[\omega] .
$$


Lemma 3. The map $\mathcal{D}$ is onto if $\operatorname{deg}(A-B)-a-b>2 g-2$.

We consider a divisor $D$ as in $(\star)$ and a section $s \in \mathcal{H}(X, D) \backslash\{0\}$ with zero divisor $\operatorname{div}(s)=E$. The multiplication by $s$ gives an isomorphism:

$$
m(s): H^{0}(X, S(D)) \rightarrow H^{0}(X, \Omega(2 D-E)) .
$$

Composing with $\mathcal{D}$ we obtain

$$
\left.\mathcal{D} \circ m(s): H^{0}(X, S(D)) \rightarrow H^{1}(X \backslash[D]), \mathbb{C}\right), \quad v \mapsto[s v],
$$

which is $d \Upsilon(s)$ (up to a constant).

We would like to apply Lemma 3 We first decompose the divisor of $s$ into the sum of two effective divisors as follows:

$$
E=E_{1}+E_{2} \text { with }[D]-\left[E_{1}\right] \geq 0,\left[E_{2}\right] \cap[D]=\emptyset .
$$

Let us define

$$
A=2 D-E_{1}, B=E_{2} .
$$

With this choice, Lemma 3 gives the surjectivity of $d \Upsilon(s)$ if the couple $(D, E)$ satisfies

a) $2 D-E_{1} \geq 0$,

b) $\operatorname{deg}(2 D-E)-k-\operatorname{deg}\left[E_{2}\right]>2 g-2$.

Notation 3. Let $T \in X \backslash[D]$ be a generic point and $r \geq 1$ be an integer. Set

$$
\begin{array}{r}
\mathbb{P}(\mathcal{H}(X, D)):=\left\{(s) \in \mathbb{P} H^{0}(X, S(D)): s \in \mathcal{H}(X, D)\right\}, \\
\mathbb{P} H^{0}(X, S(D-r T)):=\left\{(s) \in \mathbb{P} H^{0}(X, S(D)): \operatorname{div}(s) \geq r T\right\} .
\end{array}
$$

Notice that

$$
\begin{aligned}
& \operatorname{dim} \mathbb{P}(\mathcal{H}(X, D)) \geq \operatorname{deg}(D)-2 g-k, \\
& \operatorname{dim} \mathbb{P} H^{0}(X, S(D-r T))=\operatorname{deg}(D)-1-r .
\end{aligned}
$$

Therefore, if $r \leq \operatorname{deg}(D)-2 g-k$, then

$$
\mathbb{P}(\mathcal{H}(X, D)) \cap \mathbb{P} H^{0}(X, S(D-r T)) \neq \emptyset .
$$

Let $s \in \mathcal{H}(X, D)$ be a nontrivial section with $\operatorname{div}(s) \geq(\operatorname{deg}(D)-2 g-k) T$. Then we have

$$
\operatorname{deg}\left(E_{1}\right) \leq(g-1+\operatorname{deg}(D))-(\operatorname{deg}(D)-2 g-k)=3 g+k-1 .
$$

In particular, a) holds for $E=\operatorname{div}(s)$ when

$$
2 n_{i}>3 g+k-1, i \in\{1, \ldots, k\} .
$$

Then b) becomes $\operatorname{deg}(D)-\operatorname{deg}\left[E_{2}\right]>3 g-3+k$. Since

$$
\operatorname{deg}\left[E_{2}\right] \leq \operatorname{deg}[E] \leq 3 g+k-1,
$$

condition b) holds when

$$
\operatorname{deg}(D)>6 g+2 k-4 .
$$

Under these hypotheses, Lemma 3 implies that all components of $\mathcal{H}(X, D)$ contain a point $s$ such that $d \Upsilon(s)$ is surjective. The implicit function theorem finally gives:

Proposition 2. Let $X$ be a compact Riemann surface of genus $g$ and $D$ be a divisor as in $(\star)$ with support of degree $k$. Suppose that

1) $2 n_{i}>3 g+k-1, i \in\{1, \ldots, k\}$,

2) $\operatorname{deg}(D)>6 g+2 k-4$. 
Then the general point of any component of $\mathcal{H}(X, D)$ is smooth and

$$
\operatorname{dim} \mathcal{H}(X, D)=\operatorname{deg}(D)-2 g-k+1 .
$$

\section{Constructing maps With even monodromy}

In the previous section we have seen that sections in $\mathcal{H}(X, D)$ give in a natural way odd ramification coverings. However, Proposition 2 only gives a bound on the ramification at infinity of such maps. In fact, "a priori" these sections could have zeros at $D$; hence the degree of the corresponding maps could drop. Moreover, we need to provide indecomposable maps.

Let $D=n_{1} P_{1}+n_{2} P_{2}+n_{3} P_{3}$ as in the previous sections with $k$ the cardinality of its support. Set

$$
d_{1}=2 n_{1}-1, d_{2}=2 n_{2}-1, d_{3}=2 n_{3}-1 .
$$

Definition 3. We will say that $\left(d_{1}, d_{2}, d_{3}\right)$ is an indecomposable triple in these cases:

- if $k=1: d_{1}$ is a prime number;

- if $k=2: d_{1}, d_{2}$ are relatively prime;

- if $k=3: d_{1}, d_{2}, d_{3}$ are relatively prime.

The result is the following:

Proposition 3. Let $X$ be a compact Riemann surface of genus $g>0$ and $D$ be a divisor as in $(\star)$ with support of degree $1 \leq k \leq 3$. Let $d_{1}, d_{2}$ and $d_{3}$ be as before. Suppose

a) $d_{i}>3 g+k$ for all $i \in\{1, \ldots, k\}$;

b) $\operatorname{deg}(D)>6 g+2 k-3$;

c) $\left(d_{1}, d_{2}, d_{3}\right)$ is an indecomposable triple.

Then there exists a family $\mathcal{F}(X, D)$ of meromorphic functions $f$ on $X$ with these properties:

1) $\operatorname{deg}(f)=2 \operatorname{deg}(D)-k$;

2) $M(f) \subset A_{d}$ and $M(f)$ is primitive, i.e. $f$ is indecomposable;

3) $f$ has maximum ramification in $[D]$.

Moreover, $\operatorname{dim} \mathcal{F}(X, D)=\operatorname{deg}(D)-2 g-k+2$.

Proof. By construction the divisors

$$
D_{i}=D-P_{i}, i \in\{1, \ldots, k\},
$$

satisfy the hypothesis of Proposition 2, Then we get

$$
\operatorname{dim} \mathcal{H}\left(X, D_{i}\right)=\operatorname{deg}(D)-2 g-k=\operatorname{dim} \mathcal{H}(X, D)-1, \quad i \in\{1, \ldots, k\} .
$$

Comparing dimensions, we get the existence of sections $s$ in

$$
\mathcal{H}(X, D) \backslash \bigcup_{i=1}^{k} \mathcal{H}\left(X, D_{i}\right) .
$$

They have the property:

$$
\operatorname{ord}_{P_{i}}\left(s^{2}\right)=-2 n_{i}, i \in\{1, \ldots, k\} .
$$

This proves the existence of a family of odd ramification coverings $\mathcal{F}(X, D)$ with maximum ramification in the points of $[D]$. Notice that maps in $\mathcal{F}(X, D)$ have degree

$$
d=2 \operatorname{deg}(D)-k .
$$


Moreover,

$$
\operatorname{dim} \mathcal{F}(X, D)=\operatorname{deg}(D)-2 g-k+2 .
$$

Finally, condition c) and Lemma 2 give indecomposability of maps in $\mathcal{F}(X, D)$.

Proposition 3 specializes to three cases as follows:

- $k=1$ : we get families of indecomposable odd ramification coverings with a pole $P_{1}$ of total ramification and prime degree $d$.

- $k=2$ : we get families of indecomposable odd ramification coverings of (even) degree $d=2 n_{1}+2 n_{2}-2$ and ramification $d_{1}=2 n_{1}-1$ at $P_{1}$, $d_{2}=2 n_{2}-1$ at $P_{2}$ with $d_{1}$ and $d_{2}$ relatively prime.

- $k=3$ : we get families of indecomposable odd ramification coverings of (odd) degree $d=2 n_{1}+2 n_{2}+2 n_{3}-3$ and ramification $d_{1}=2 n_{1}-1$ at $P_{1}$, $d_{2}=2 n_{2}-1$ at $P_{2}, d_{3}=2 n_{3}-1$ at $P_{3}$ with $d_{1}, d_{2}, d_{3}$ relatively prime.

Notice that condition $b$ ) in Proposition 3 implies that

$$
d>12 g+3 k-6 .
$$

It can be easily proved that, for every integer $d \geq 12 g+4$, there exists a divisor $D$ in the hypotheses of Proposition 3 with $d=2 \operatorname{deg}(D)-k$. For $d$ a fixed positive integer, we use the following notation:

$$
\mathcal{F}(X, d)=\bigcup_{D \in \mathcal{D}(d)} \mathcal{F}(X, D)
$$

where $\mathcal{D}(d)=\{D \in \operatorname{Div}(X): D$ as in Proposition $3 k>1,2 \operatorname{deg}(D)-k=d\}$. Notice that, for every $D \in \mathcal{D}(d)$ :

$$
\operatorname{dim} \mathcal{F}(X, d)=\operatorname{deg}(D)-2 g+2=\left[\frac{d+3}{2}\right]-2 g+2 .
$$

Proof of Theorem 1. Notice that Proposition 3 gives a weak version of Theorem 1. In fact, we need to show that the generic map in $\mathcal{F}(X, d)$ has monodromy group equal to $A_{d}$. By Lemma 1 it is enough to prove that its monodromy group contains at least a 3 -cycle.

Let $d$ be an even integer. Suppose, by contradiction, that the monodromy group of a generic map $f \in \mathcal{F}(X, d)$ does not contain any 3-cycle. It follows that any branch point has ramification order $\geq 4$. Then Hurwitz's formula gives

$$
2 g-2=-2 d+(d-2)+\sum_{P \in X}\left(e_{f}(P)-1\right) \geq-d-6+4 b
$$

where $b$ is the number of branch points. Thus, $b \leq(2 g+4+d) / 4$. Then, from the theory of Hurwitz schemes,

$$
\operatorname{dim} \mathcal{F}(X, d) \leq(2 g-4+d) / 4
$$

On the other hand,

$$
\operatorname{dim} \mathcal{F}(X, d)=d / 2-2 g+2>(2 g-4+d) / 4 \text { if } d>10 g-12 .
$$

Similar considerations hold for odd $d$. 
Remark 1. Let $\mathcal{F}_{1}(d)$ be the union of all families $\mathcal{F}(X, D)$ with $k=1,2 \operatorname{deg}(D)-1=$ $d$ and $X$ moving in the moduli space of smooth projective curves $\mathcal{M}_{g}$. A count of parameters shows that the generic element in $\mathcal{F}_{1}(d)$ is a couple $(X, f)$ where $f$ has total ramification at infinity and all other ramification points of index 3 with distinct images in $\mathbb{P}^{1}$.

\section{SimPLE ODD RAMIFICATIONS}

Let $X$ be a compact Riemann surface of genus $g$ and $f: X \rightarrow \mathbb{P}^{1}$ a meromorphic function with branch locus $B$ and ramification locus $R$. The simplest odd ramification type is a $3: 1$ ramification covering (see [7]).

Definition 4. We say that an odd ramification covering is simple if all points in $R$ have ramification index equal to 3 and have distinct images in $\mathbb{P}^{1}$.

Using admissible coverings we prove:

Proposition 4. 1) Any odd ramification covering $f: X \rightarrow \mathbb{P}^{1}$ is the specialization of a family of simple odd ramification coverings $f_{s}: X_{s} \rightarrow \mathbb{P}^{1}$.

2) The monodromy group of $f$ is a subgroup of the monodromy group of $f_{s}$ for general s.

Notation 4. Set $Y_{0}=\mathbb{P}^{1}$, and let $T \in Y_{0}$ be a branch point of $f$. Let $Y_{1}$ be another copy of $\mathbb{P}^{1}$ and $P$ a point in $Y_{1}$. We call $\tilde{Y}$ the singular rational curve obtained by gluing $Y_{1}$ and $Y_{0}$ in $T$ and $P: \tilde{Y}=Y_{0} \cup Y_{1}$. Let $D=f^{-1}(T)=\sum_{i=1}^{r}\left(2 n_{i}-1\right) P_{i}$. For any $i \in\{1, \ldots, r\}$ we take a copy $X_{i}$ of $\mathbb{P}^{1}$ with a selected point $Q_{i} \in X_{i}$. Let

$$
\tilde{X}=\left(\bigcup_{i=1}^{r} X_{i}\right) \cup X
$$

be the curve obtained by identifying $P_{i}$ and $Q_{i}$ for every $i \in\{1, \ldots, r\}$.

Lemma 4. We can define a map $\tilde{f}: \tilde{X} \rightarrow \tilde{Y}$ satisfying the following properties:

a) $\left.\tilde{f}\right|_{X}=f$

b) $\left.\tilde{f}\right|_{X_{i}}=f_{i}: X_{i} \rightarrow Y_{1}$ for every $i \in\{1, \ldots, r\}$,

c) $f_{i}$ is an odd ramification covering of degree $2 n_{i}-1$ with total ramification at $Q_{i}$,

d) ramification points of $f_{i}$ other than $Q_{i}$ have index 3 ,

e) branch points of the $f_{i}$ other than $T$ are all distinct.

Proof. The existence of meromorphic functions $X_{i} \rightarrow \mathbb{P}^{1}$ satisfying c), d) and e) follows from Riemann's existence theorem.

Notice that we have constructed an admissible covering. Now we can apply the standard smoothing procedure (see [15] and [1]).

Proof of Proposition 4. We can consider $\tilde{Y}$ as the union of two lines in the projective plane: $\tilde{Y}=\{x y=0\}$. Choose local coordinates $w_{i}$ and $z_{i}$ at $Q_{i}=P_{i}$ such that locally $\tilde{X}$ is given by $\left\{w_{i} z_{i}=0\right\}$ and $\tilde{f}$ has the form $x=w_{i}^{2 n_{i}-1}$ on $X$ and $y=z_{i}^{2 n_{i}-1}$ on $X_{i}$ for each $i \in\{1, \ldots, r\}$. Now set $m_{i}=2 n_{i}-1$ for $i=1, \ldots, r$. Let $\Delta$ be the curve in $\mathbb{C}^{r+1}$ defined by

$$
\Delta=\left\{\left(t, t_{1}, \ldots, t_{r}\right): t=t_{i}^{m_{i}},|t|<1,\left|t_{i}\right|<1, i=1, \ldots, r\right\} .
$$


For every $s=\left(t, t_{1}, \ldots, t_{r}\right) \in \Delta$, consider

$$
\begin{aligned}
I_{s} & =\{(x, 0):|x| \leq|t(s)|\} \cup\{(0, y):|y| \leq|t(s)|\} \subset \tilde{Y}, \\
J_{i, s} & =\left\{\left(w_{i}, 0\right):\left|w_{i}\right| \leq\left|t_{i}(s)\right|\right\} \cup\left\{\left(0, z_{i}, s\right):\left|z_{i}\right| \leq\left|t_{i}(s)\right|\right\} \subset \tilde{X} .
\end{aligned}
$$

Let $U_{s}=\tilde{Y} \backslash I_{s}$ and $U_{s}^{\prime}=\tilde{X} \backslash \bigcup_{i} J_{i, s}$. Finally, we define

$$
\begin{gathered}
V_{s}=\left\{(x, y) \in \mathbb{C}^{2}: x y=t(s),|x|<1,|y|<1\right\}, \\
V_{i, s}=\left\{\left(w_{i}, z_{i}\right) \in \mathbb{C}^{2}: w_{i} z_{i}=t_{i}(s),\left|w_{i}\right|<1,\left|z_{i}\right|<1\right\} .
\end{gathered}
$$

We smooth locally $\tilde{Y}$ by gluing $U_{s}$ to $V_{s}$ in the following way:

$$
(x, 0) \mapsto\left(x, \frac{t(s)}{x}\right) ;(0, y) \mapsto\left(\frac{t(s)}{y}, y\right) .
$$

We can do the same for $\tilde{X}$ by gluing $U_{s}^{\prime}$ to $V_{i, s}$ :

$$
\left(w_{i}, 0\right) \mapsto\left(w_{i}, \frac{t_{i}(s)}{w_{i}}\right) ;\left(0, z_{i}\right) \mapsto\left(\frac{t_{i}(s)}{z_{i}}, z_{i}\right) .
$$

For each $s \in \Delta$ we get a compact smooth surface $X_{s}$ and a copy $Y_{s}$ of $\mathbb{P}^{1}$. We can define a map $f_{s}: X_{s} \rightarrow Y_{s}$ with $f_{s}=\tilde{f}$ on $U_{s}^{\prime}$ and $f_{s} \mid V_{i, s}$ given by

$$
\left(w_{i}, z_{i}\right) \mapsto\left(w_{i}^{m_{i}}, z_{i}^{m_{i}}\right) .
$$

In fact, we get compact Riemann surfaces $X_{s}$ (the complex structure on $X_{s}$ is given by the ramified covering) of genus $g$ and odd ramification coverings $f_{s}$ on $X_{s}$ with the same degree of $f$ that specializes to $\tilde{f}$ for $s=0$. Remark that the monodromy group of $f$ is determined by the branches in $Y_{0} \backslash\{T\}$. Let $\gamma_{1}, \ldots, \gamma_{b}$ be loops in $Y_{0}$ corresponding to these branches. Since the coverings $f_{s}$ and $f$ are topologically the same in $U_{s}^{\prime}$, we can find $\gamma_{1, s}, \ldots, \gamma_{b, s}$ loops in $Y_{s} \backslash V_{s}$ giving the same monodromy as $\gamma_{1}, \ldots, \gamma_{b}$. Then, the monodromy group $M\left(f_{s}\right)$ contains $M(f)$ as a subgroup for every $s \in \Delta$. Moreover, the coverings $f_{s}$ have simpler odd ramification. Repeating this procedure for each branch point we finally get a family of simple odd ramification coverings deforming $f$.

Remark 2. It is not a trivial problem to find a deformation of $f$ as in Proposition 4 that preserves the conformal structure of $X$.

Combined with Theorem 1 this implies:

Theorem 2. The generic Riemann surface of genus $g$ admits simple odd ramification coverings of degree $d \geq 12 g+4$ with monodromy group equal to $A_{d}$.

\section{A technical Result}

Let $A, B \in \operatorname{Div}(X)$ be two effective divisors on $X$ with $[A] \cap[B]=\emptyset, a=\operatorname{deg}[A]$, $b=\operatorname{deg}[B]$. Let us call $A^{\prime}=A-[A]$. We define a sheaf $\mathcal{R}=\mathcal{R}(A, B)$ as follows:

$$
\mathcal{R}(A, B)(U):=\left\{f \in \mathcal{O}\left(A^{\prime}\right)(U): d f \in \Omega(-B)(U)\right\},
$$

$U \subset X$ an open set. We have the short exact sequence of sheaves:

$$
0 \rightarrow \mathcal{O}\left(A^{\prime}-B-[B]\right) \stackrel{i}{\rightarrow} \mathcal{R} \stackrel{e_{v}}{\rightarrow} \mathbb{C}_{B} \rightarrow 0
$$


where $\mathbb{C}_{B}$ is the skyscraper sheaf with support in $[B]$ and $e_{v}$ is the "valuation" morphism. If $f \in \mathcal{R}(A, B)(U)$, then $d f \in \Omega(A-B)(U)$, and a second exact sequence is then

$$
0 \rightarrow \mathbb{C} \stackrel{i}{\rightarrow} \mathcal{R} \stackrel{d}{\rightarrow} \Omega(A-B)^{\circ} \rightarrow 0
$$

where $\Omega(A-B)^{\circ}$ is the subsheaf of forms with residues zero in $[A]$. Finally, taking residues, we get a third sequence:

$$
0 \rightarrow \Omega(A-B)^{\circ} \rightarrow \Omega(A-B) \stackrel{\text { res }}{\rightarrow} \mathbb{C}_{A} \rightarrow 0 .
$$

Consider the coboundary operators associated to sequence (2):

$$
\Delta_{i}: H^{i-1}\left(X, \Omega(A-B)^{\circ}\right) \rightarrow H^{i}(X, \mathbb{C}), \quad i=1,2 .
$$

Suppose now that $H^{1}(X, \mathcal{R})=0$. Then $\Delta_{1}$ is surjective and $\Delta_{2}$ is an isomorphism. Moreover, the image of the map res : $H^{0}(X, \Omega(A-B)) \rightarrow H^{0}\left(X, \mathbb{C}_{A}\right)=\mathbb{C}^{a}$ is contained in $\mathcal{I}=\left\{\sum_{1}^{a} x_{i}=0\right\}$ by the residue theorem. Sequence (3) then gives $H^{1}(X, \Omega(A-B))=0$. Therefore $\Delta_{1}$ and res are both surjective. Finally, this implies the surjectivity of the De Rham map:

$$
\mathcal{D}: H^{0}(X, \Omega(A-B)) \rightarrow H^{1}(X \backslash[A], \mathbb{C}), \quad \omega \mapsto[\omega] .
$$

Proposition 5. If $H^{1}(X, \mathcal{R})=0$, then the map $\mathcal{D}$ is surjective.

In fact, the vanishing of $H^{1}(X, \mathcal{R})$ and the surjectivity of $\mathcal{D}$ are equivalent conditions (see [19]).

Proof of Lemma[3. We have $H^{1}\left(X, \mathbb{C}_{B}\right)=0$ and $H^{1}\left(X, \mathcal{O}\left(A^{\prime}-B-[B]\right)=0\right.$. This implies that $H^{1}(X, \mathcal{R})=0$.

\section{ACKNOWLEDGEMENTS}

We would like to thank the referee and Michael D. Fried for valuable suggestions. We are grateful to Juan Carlos Naranjo and Enrico Schlesinger for many helpful conversations.

\section{REFERENCES}

1. E. Arbarello, M. Cornalba, P. Griffiths, J. Harris. Geometry of algebraic curves I, Grundlehren Math. Wiss. (267) Springer, Berlin, 1985. MR0770932 (86h:14019)

2. P. Bailey and M. Fried. Hurwitz monodromy, spin separation and higher levels of a modular tower, Arithmetic fundamental groups and noncommutative algebra, editors M. Fried and Y. Ihara, 79-220, Proc. Sympos. Pure Math., (70) Providence, RI, 2002. MR1935406

3. S. Brivio and G. Pirola. Alternating groups and rational functions on surfaces, preprint.

4. M. Cornalba. Systèmes pluricanoniques sur l'espace des modules des courbes et diviseurs de courbes k-gonales (d'après Harris et Mumford), Seminar Bourbaki, vol.1983/84, Astérisque No. 121-122, 7-24, 1985. MR0768951 (86c:14021)

5. M. Fried. Combinatorial computation of moduli dimension of Nielsen classes of covers, Contemp. Math., (89), 61-79, Amer. Math. Soc., Providence, RI. 1989. MR1006477 (90j:12007)

6. M. Fried. Introduction to modular towers: generalizing dihedral group-modular curve connections, Recent developments of the inverse Galois problem, Contemp. Math., (186), 111-171, Amer. Math. Soc., Providence, RI. 1995. MR.1352270 (97a:11070)

7. M. Fried. Alternating Group and Lifting Invariants, (preprint)

8. W. Fulton. Hurwitz schemes and irreducibility of moduli of algebraic curves, Ann. Math. 90, 542-575, 1969. MR0260752 (41:5375)

9. R. Guralnick. Monodromy groups of coverings of curves, Galois Groups and Fundamental Groups. Leila Schneps Ed., Cambridge Univ. Press, Cambridge, 2003. MR2012212 
10. R. Guralnick and K. Magaard. On the minimal degree of a primitive permutation group, J. Algebra, 207, no.1, 127-145, 1998. MR1643074 (99g:20014)

11. R. Guralnick and M. Neubauer. Monodromy groups of branched coverings: the generic case, Recent developments of the inverse Galois problem (Seattle, Wa, 1993), Contemp. Math., (186), 325-352, Amer. Math. Soc., Providence, RI, 1995. MR,1352281 (96h:20007)

12. R. Guralnick and J. Shareshian. Symmetric and Alternating Groups as Monodromy Groups of Riemann Surfaces I: Generic Covers and Covers with Many Branch Points, preprint.

13. R. Guralnick and J. Thompson. Finite groups of genus zero, J. Algebra, (131), 303-341, 1990. MR 1055011 (91e:20006)

14. J. Harris. Galois groups and enumerative problems, Duke Math. J., 46 (4), 624-724, 1979. MR0552521 (80m:14038)

15. J. Harris and D. Mumford. On the Kodaira dimension of the moduli space of curves, Invent. Math. (67), 23-88, 1982. MR.0664324 (83i:14018)

16. S. Kleiman and D. Laksov. Another proof of the existence of special divisors, Acta Math., (132), 163-175, 1974. MR0357398 (50:9866)

17. K. Magaard and H. Völklein. The monodromy group of a function on a general curve, Israel J. Math., to appear.

18. R. Miranda. Algebraic Curves and Riemann Surfaces. Grad. Stud. in Math. 5, Amer. Math. Soc., Providence, RI, 1995. MR1326604 (96f:14029)

19. G. Pirola. Algebraic curves and non-rigid minimal surfaces in the Euclidean space, Pacific J. Math., (183), no. 2, 333-357, 1998. MR1625966 (99e:53009)

20. J. P. Serre. Relèvement dans $\tilde{\mathcal{A}}_{n}$, C. R. Acad. Sci. Paris (311), no. 8, 477-482, 1990. MR 1076476 (91m:20010)

21. J. P. Serre. Revêtements à ramification impaire and thêta caratéristiques, C. R. Acad. Sci. Paris (311), no. 9, 547-552, 1990. MR1078120 (92a:14022)

22. O. Zariski. Collected Papers Vol. III, MIT Press, Cambridge, MA, 1972-1979.

Dipartimento di Matematica "DimA", Universitá di Genova, via Dodecaneso 35, 16146 Genova, Italia

Current address: Dipartimento di Matematica "F. Casorati", Universitá di Pavia, via Ferrata 1, 27100 Pavia, Italia

E-mail address: artebani@dimat.unipv.it

Dipartimento di Matematica "F. Casorati", Universitá di Pavia, via Ferrata 1, 27100 Pavia, Italia

E-mail address: pirola@dimat.unipv.it 